\title{
Degradation of Structurally Defined Graphene Nanoribbons by Myeloperoxidase and Photo-Fenton Reaction
}

Xiangfeng Luan, ${ }^{a}$ Cristina Martín, ${ }^{b}$ Pengfei Zhang, ${ }^{a}$ Qian Li, ${ }^{a}$ Isabella Anna Vacchi ${ }^{b}$ Lucia Gemma Delogu ${ }^{c}$ Yiyong Mai, ${ }^{* a}$ and Alberto Bianco ${ }^{* b}$

X. Luan, P. Zhang, Q. Li, Prof. Y. Mai

School of Chemistry and Chemical Engineering, Frontiers Science Center for Transformative Molecules, Shanghai Key Laboratory of Electrical Insulation and Thermal Ageing, Shanghai Jiao Tong University, 800 Dongchuan Road, Shanghai 200240, China

E-mail: mai@sjtu.edu.cn

Dr. C. Martin, Dr. I. A. Vacchi, Dr. A. Bianco

CNRS, Immunology, Immunopathology and Therapeutic Chemistry, UPR 3572, University of Strasbourg, ISIS, Strasbourg 67000, France

Email: a.bianco@ibmc-cnrs.unistra.fr

Prof. L. G. Delogu

Department of Biomedical Sciences, University of Padua, Padova 35121, Italy

Keywords: Carbon materials; Functionalization, Biodegradability; Peroxidases, Hydroxyl radicals

Abstract: As an emerging member of the graphene family, structurally defined graphene nanoribbons (GNRs) have shown promising applications in various fields. The evaluation of the degradability of GNRs is particularly important for assessing the persistence level and risk of these materials in living organisms and the environment. However, there is a void in the study of the degradation of GNRs. Here, we report the degradation behavior of GNRs in the presence of human myeloperoxidase (hMPO) or treated with photoFenton $(\mathrm{PF})$ reaction. With the assistance of potassium hydroxide or imidazole, which facilitates the dispersion of GNRs in the aqueous solution, GNRs underwent only partial degradation after 25-hour incubation with hMPO, while, the PF reaction degraded GNRs almost completely after 120 hours. These results indicate that structurally precise GNRs can be efficiently degraded under suitable conditions, providing more opportunities for future applications in different fields. 
In recent years, graphene and its derivatives have been widely studied in the fields of medicine and gene delivery, bioimaging, biodetection, immune-modulation and cancer treatment. ${ }^{[1-4]}$ As an emerging member in the graphene family, structurally well-defined graphene nanoribbons (GNRs) have attracted enormous attention due to their controllable structures including length, width and edges. ${ }^{[5-19]}$ GNRs have proven great potential in various applications such as photothermal therapy, super-resolution imaging and semiconductor materials. For instance, Mai and colleagues ${ }^{[20]}$ synthesized arm-chair edged GNRs with a uniform width of $1.7 \mathrm{~nm}$, which were functionalized with hydrophilic polyethylene oxide (PEO). These GNRs showed excellent dispersibility in aqueous phase and strong absorption in the near-infrared (NIR) region. Meanwhile, these GNRs show excellent photothermal conversion efficiency. ${ }^{[21]}$ Through the cooperative self-assembly of these GNRs with a cationic porphyrin (Pp4N) in aqueous solution, the resulting supramolecular GNR-Pp4N hybrid nanowires show synergistic photodynamic and photothermal dual-modal therapeutic capability, which enable the elimination of a wide spectrum of Gram-positive, Gram-negative, and drug-resistant bacteria. ${ }^{[22]}$ As another typical example, Fischer's group introduced Cy5 fluorescent molecules to the edge of cove-type GNRs with a uniform width of $1 \mathrm{~nm} .{ }^{[23]}$ Their encouraging results revealed that GNRs can behave as a flexible fluorescent carrier, allowing fluorescence imaging in living organisms and various other environments. On the other hand, as the nextgeneration semiconductor materials, the applications of GNRs in the field effect transistor, ${ }^{[24-26]}$ electrocatalytic $\mathrm{CO}_{2}$ reduction, ${ }^{[27]}$ quantum spintronic, ${ }^{[28]}$ gas sensing ${ }^{[29-}$ ${ }^{31]}$ and electroluminescence devices, ${ }^{[32]}$ among others, ${ }^{[33-37]}$ have also been increasingly explored. 
Although GNRs show promising applications in various fields, their accumulation in living organisms and the environment has remained an unsolved problem. In this context, it is of fundamental importance to explore the degradation performance of GNRs. So far, oxidative enzymes, mainly including horseradish peroxidase (HRP) ${ }^{[38]}$ human myeloperoxidase (hMPO), ${ }^{[39,40]}$ eosinophil peroxidase (EPO), ${ }^{[1,42]}$ and lignin peroxidase $(\mathrm{LiP}),{ }^{[43]}$ have proven to be efficient agents for the biodegradation of carbon nanomaterials. In particular, hMPO is a heme protease secreted by various primary cells in the innate immune system and can be extracted from neutrophils. Compared with other peroxidases, hMPO can produce stronger oxidant $\mathrm{HClO}$ in addition to active radical intermediates during the catalytic process, which contribute to the efficient degradation of the materials. ${ }^{[40]}$ In addition to the enzyme-catalyzed biodegradation, photo-Fenton (PF) reaction has also proven to be efficient for the degradation of various nanomaterials. ${ }^{[44,45]}$ In acidic solutions, $\mathrm{Fe}^{3+}$ mainly exists as $\mathrm{Fe}(\mathrm{OH})^{2+}$, and can effectively absorb the ultraviolet light. Upon UV irradiation, $\mathrm{Fe}(\mathrm{OH})^{2+}$ ions undergo a photoreduction, producing $\mathrm{Fe}^{2+}$ and hydroxyl radicals. Next, additional quantities of highly reactive $\mathrm{HO}^{\circ}$ are produced by Haber-Weiss reaction between $\mathrm{Fe}^{2+}$ and $\mathrm{H}_{2} \mathrm{O}_{2}$. Compared to the traditional Fenton reaction, the assistance of ultraviolet light accelerates the production of a large amount of $\mathrm{HO}^{\circ}$, and the degradation rate is greatly increased. ${ }^{[46]}$ The powerful degradation action of PF reaction depends on the high electronegativity of the hydroxyl radical (its electron affinity is $569.3 \mathrm{~kJ}$ and the oxidation potential is up to $+2.31 \mathrm{~V}) .{ }^{[47]}$ This oxidative environment can even completely degrade hexagonal boron nitride nanosheets with high oxidation resistance. ${ }^{[48]}$ Overall, hMPO-catalyzed degradation may provide a new method for safe degradation of nanomaterials in some biological environments, while the photo-Fenton reaction with strong oxidative capacity can reduce the toxicity and accumulation of waste materials exposed to the environment. 
By taking advantage of these two degradation protocols, we studied the degradation performance of structurally defined GNRs and evaluated their persistence level under different oxidation conditions. Meanwhile, the significant effect of the dispersibility of GNRs on their degradation performance was also unveiled. Our study provides effective approaches and valuable information for the degradation of structurally defined GNRs, affording more possibilities for further exploration of their applications.

To evaluate the degradability of GNRs, we employed carboxyl-functionalized graphene nanoribbons (GNR-COOH) with an armchair edge structure, a backbone width of $1.7 \mathrm{~nm}$ and an average length of $15 \mathrm{~nm}$, synthesized following a previous protocol. ${ }^{[20]}$

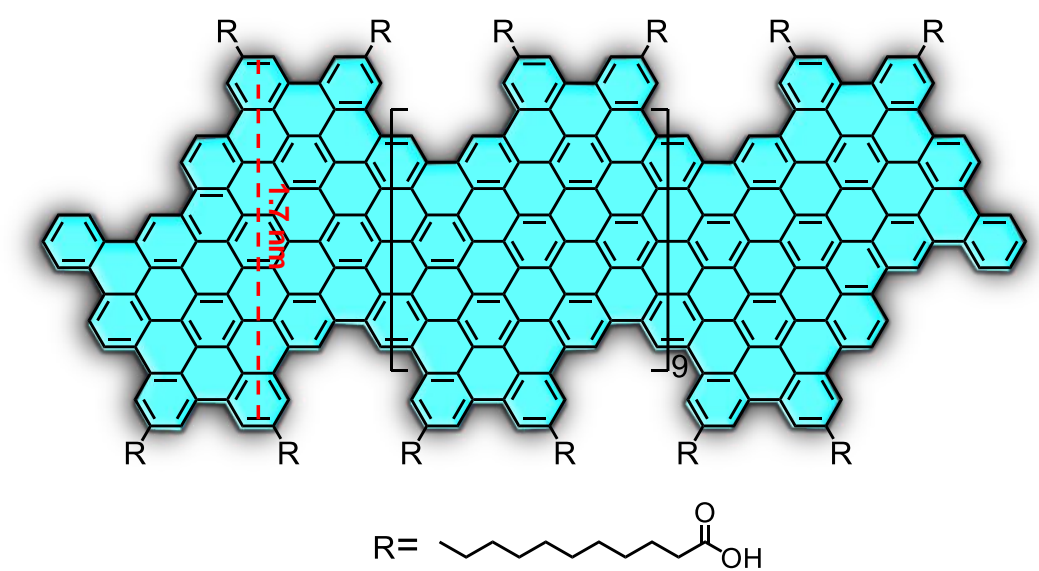

Figure 1. Molecular structure of the structurally defined GNR-COOH

First, we selected hMPO to evaluate the biodegradation behavior of the GNRs. The GNRs were directly dispersed in the aqueous phase at a concentration of $14 \mu \mathrm{g} \cdot \mathrm{mL}^{-1}$ through strong tip sonication for 30 minutes (see experimental section in the Supporting Information). Then, the pre-dispersed GNR samples were incubated with hMPO in phosphate buffer solutions containing $140 \mathrm{mM} \mathrm{NaCl}$. In the process of degradation, hydrogen peroxide was added to the GNRs dispersion every one hour while hMPO was replenished every five hours. The GNRs in their dispersions at the beginning of the degradation $(\mathrm{t}=0 \mathrm{~h})$ were considered as control samples. 


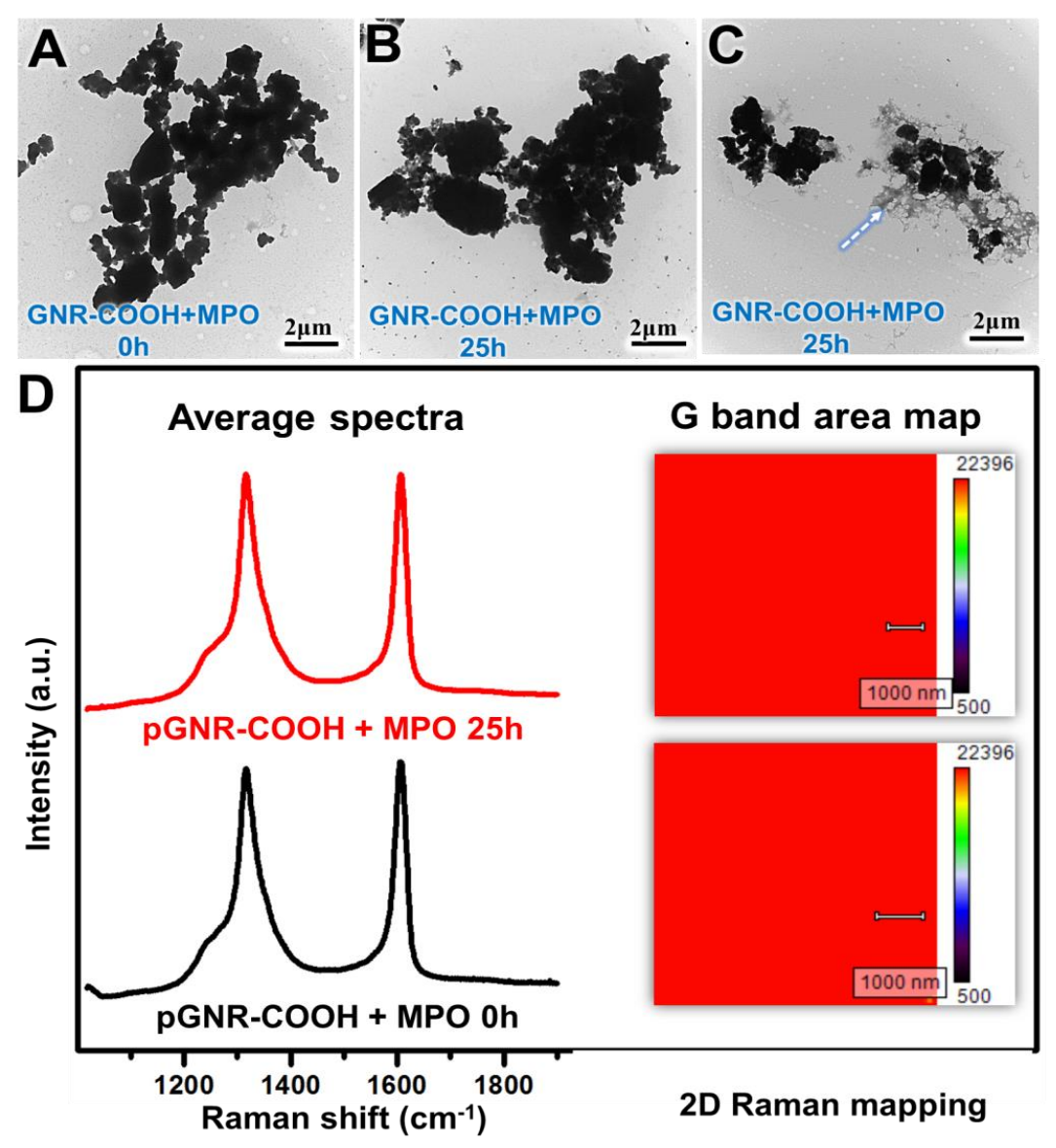

Figure 2. TEM images of GNR-COOH dispersed in phosphate buffer solution: after treatment with hMPO for $(\mathrm{A}) \mathrm{t}=0 \mathrm{~h}$ (control); $(\mathrm{B}, \mathrm{C}) \mathrm{t}=25 \mathrm{~h}$ (the white dotted arrow points to a slightly degraded area). (D) Average Raman spectra (left side) and G band area 2D mapping images (right side) of the GNR samples. The color bar intensity (which quantifies $\mathrm{G}$ band area) does not change due to the complete homogeneity.

The morphology of the GNRs treated with hMPO was investigated by transmission electron microscopy (TEM) and Raman spectroscopy. For the control sample $(\mathrm{t}=0 \mathrm{~h})$, TEM images reveal the formation of large irregular aggregates in phosphate buffer (Figure 2A), which is attributed to the serious aggregation of the GNRs caused by their strong $\pi-\pi$ stacking in a solution rich in different salts. The sizes of the GNR aggregates reach the micrometer scale. After 25-hour incubation with hMPO (Figure 2B), only partial morphological changes and structural damages for the GNRs are observed. Few thinner marginal zones show detective porous features (Figure $2 \mathrm{C}$, area indicated by the white dotted arrow), indicative of a slight degradation. On the other hand, the average Raman spectra (obtained by averaging all collected points in the 2D Raman mapping 
mode) reveal that the intensity ratios of the $\mathrm{D}$ to $\mathrm{G}$ bands $\left(\mathrm{I}_{\mathrm{D}} / \mathrm{I}_{\mathrm{G}}\right)$ of the GNRs treated by hMPO and their control sample are basically identical (0.97-0.99) (Figure 2D, left side). Meanwhile, both samples showed very strong and uniform $G$ band signals and the D/G band ratios are identical in terms of the 2D mapping images (Figure 2D, right side; Figure S1). The Raman results confirm that no obvious degradation occurs for GNRs. Obviously, the strong aggregation prevents the interaction of the enzyme with the graphene ribbons and their effective degradation. This phenomenon is similar to that occurred to GO, in a previous study, which could not be degraded by hMPO either when seriously aggregated. ${ }^{[39]}$

In order to reduce the aggregation degree of GNRs in aqueous solution, we adopt the carboxyl group ionization procedure and the assistance of surfactant for the GNR dispersion. According to our previous reports, the carboxylic acids on GNRs can be ionized to $-\mathrm{COO}^{-}$in alkaline media. ${ }^{[21]}$ The electrostatic repulsion between $-\mathrm{COO}^{-}$may alleviate the $\pi-\pi$ stacking of the GNRs, thereby promoting their dispersibility in water. Alternatively, we though to the use of imidazole, as it has an anionic nature and a $\pi$ conjugated heterocyclic coplanar structure. ${ }^{[49]}$ As an ideal surfactant, the electrostatic repulsion between imidazole molecules adsorbed onto GNR surface may effectively inhibit their aggregation. In addition, imidazole could also cause deprotonation of the carboxyl group, thereby forming $-\mathrm{COO}^{-}$to further weaken the $\pi-\pi$ stacking between GNRs. These two synergistic effects ensure the effective and stable dispersion of the GNRs in the aqueous phase. Therefore, we employed potassium hydroxide or imidazole to enhance the dispersibility of our GNRs (see experimental details in the Supporting Information). The corresponding dispersed samples were named as pGNR-COOH (potassium hydroxide assisted) and iGNR-COOH (imidazole assisted), respectively. With the assistance of potassium hydroxide or imidazole for dispersion, the GNR samples 
were treated with hMPO under the experimental conditions similar to the above-discussed degradation of the GNRs in phosphate buffer solutions.
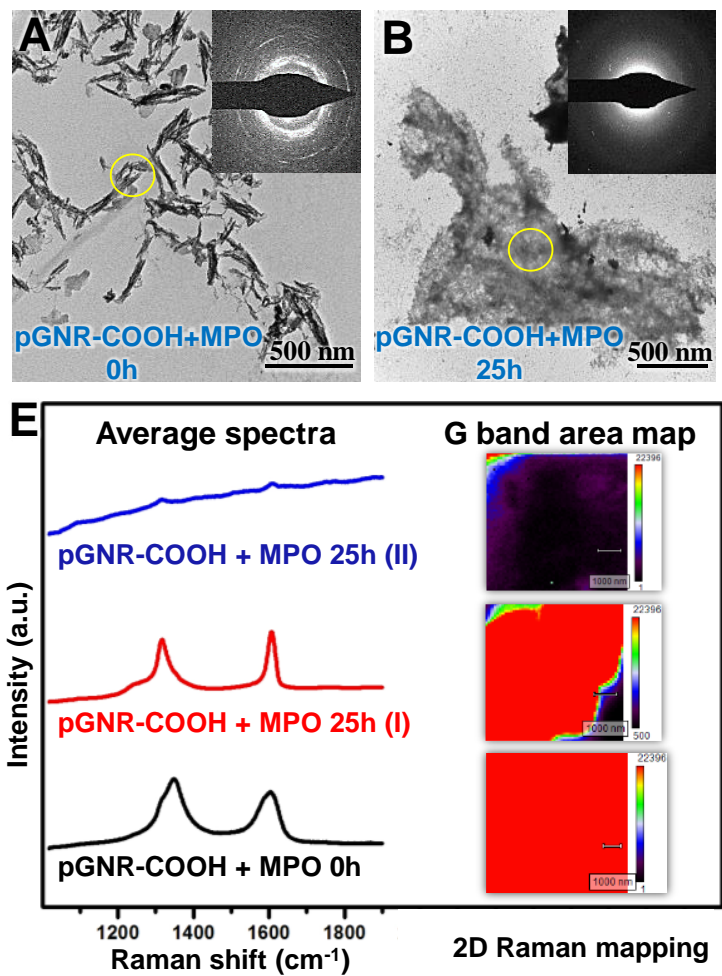

(5)
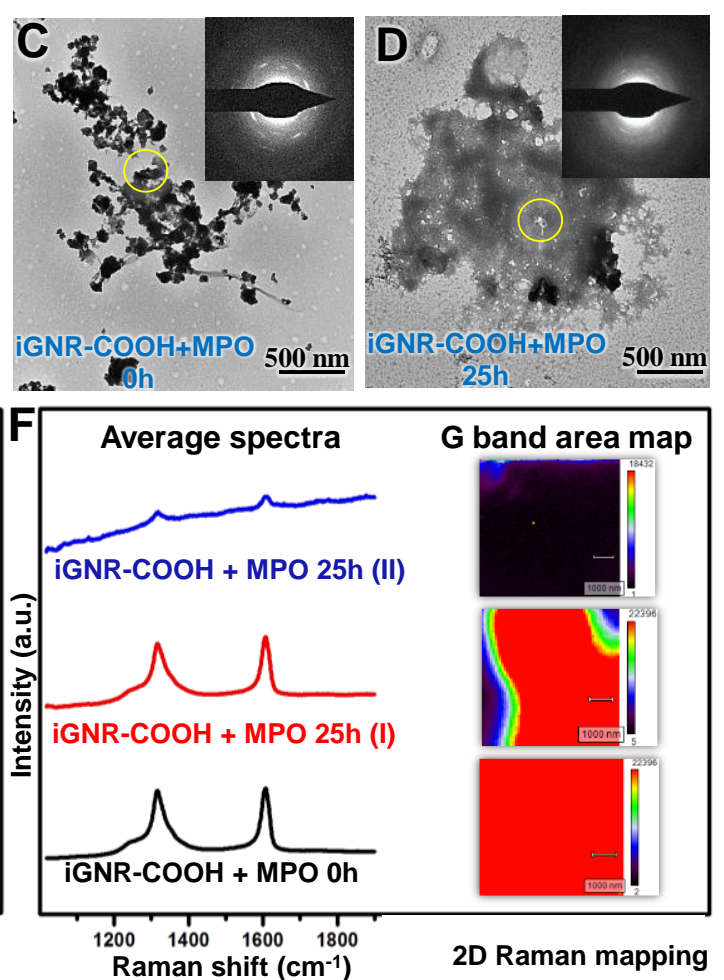

Figure 3. TEM images and the corresponding SAED patterns of pGNR-COOH (potassium hydroxide assisted) and iGNR-COOH (imidazole assisted) treated with hMPO in phosphate buffer: (A) pGNR-COOH for $0 \mathrm{~h}$ (control), (B) $25 \mathrm{~h}$; (C) iGNR$\mathrm{COOH}$ for $0 \mathrm{~h}$ (control), (D) 25 h. (E, F) Average Raman spectra (left side) and G band area $2 \mathrm{D}$ mapping (right side) of pGNR-COOH and iGNR-COOH treated with hMPO.

With the evidently improved dispersibility, pGNR-COOH shows a spindle-like structure with a much reduced average dimension compared with the GNRs without the assistance of potassium hydroxide (Figure 3A). For iGNR-COOH dispersion, nano-sized clusters were formed due to the presence of imidazole (Figure 3C). After 25-hours of incubation with hMPO in phosphate buffer solutions ( $\mathrm{pH} \sim 7.4$ ), for both samples a large number of small holes were clearly observed (Figure 3B,D), suggesting partial degradation of the GNRs in such conditions. Meanwhile, it is seen from the TEM images that the degrees of degradation for both samples are similar. The fact that obvious morphological changes were observed compared to the untreated control sample reflects 
the key roles of both hMPO and good dispersibility in the degradation of the GNRs. To further confirm the degradation, selected area electron diffraction (SAED) analyses were also used to evaluate the effects of hMPO on the crystalline patterns and graphitic structure of the GNRs. In the SAED modes (insets in Figure 3A-D), the control samples show a typical ring-like pattern with significant diffraction spots, indicative of its polycrystalline nature like graphite ${ }^{[50]}$ Nevertheless, after treated with the peroxidase for $25 \mathrm{~h}$, the diffraction signals turn very weak or disappear, indicating the loss of the polycrystalline nature and thus confirming the degradation of the GNRs.

Interestingly, in Raman analyses we obtained two types of spectra for both pGNR$\mathrm{COOH}$ and iGNR-COOH samples (Figure 3E,F, left side). The first type of spectra shows intense D and G bands, which are highly similar to those of the control samples. These Raman spectra suggest no apparent degradation of the GNRs. In sharp contrast, the other type of spectra shows almost absent $G$ and $D$ bands for both samples. Accordingly, the $G$ band area signals in the overall 2D maps also widely decreased (Figure 3E,F, right side), while in most areas we also observed a significant increase in the D/G ratio (Figure $S 2$ ). The drastic reduction of the peak intensity indicates the enzyme-catalyzed degradation of the GNRs, which damages their graphitic structure related to $\mathrm{sp}^{2}$ hybrid carbon system. ${ }^{[51]}$ However, the residual small D and G bands also suggest an incomplete degradation of the GNRs. Overall, the Raman results support the TEM observations. They demonstrate the partial degradation of the GNRs by hMPO under the improved GNRs dispersibility conditions.

GNRs inevitably enter into the environment during their applications, and their longterm accumulation and environmental exposure will cause a certain extent of pollutions. In addition to the hMPO-catalyzed degradation, PF reaction is a highly efficient method for degrading graphene materials. Herein, we also performed powerful UV-assisted PF 
reaction to evaluate the GNR degradation performance. For the GNR-COOH dispersions without the assistance of surfactants or potassium hydroxide, we found that the PF reaction could only slightly degrade the peripheral areas of the GNRs, similarly to the behavior of hMPO-catalyzed degradation (Figure S3). Therefore, we focused on the study of the effect of PF reaction on the pGNR-COOH and iGNR-COOH samples with much improved dispersibility in aqueous solutions. The PF reaction was performed in a sealed quartz bottle containing $\mathrm{FeCl}_{3}$ and $\mathrm{H}_{2} \mathrm{O}_{2}$. The GNRs dispersions were continuously irradiated by UV light with $\lambda=365 \mathrm{~nm}$ for 120 hours, with their $\mathrm{pH}$ values maintained at 4. Due to the acidic incubation medium, the spindle-like morphology of pGNR-COOH disappeared. Meanwhile, some small clusters emerged. However, the size of these clusters was much smaller than those in the control samples without the potassium hydroxide treatment. The assistance of potassium hydroxide effectively reduced the aggregation of the GNRs. Both pGNR-COOH and iGNR-COOH appeared as separate small islands with dimensions of $100-500 \mathrm{~nm}$ before the UV light irradiation $(\mathrm{t}=0 \mathrm{~h}$, Figure 4A,D). After UV irradiation for $25 \mathrm{~h}$, most of the GNR aggregates were severely damaged and showed a highly porous morphology (Figure 4B,E). In sharp contrast to the small holes in the hMPO-treated GNRs, large holes with sizes of several hundred nanometers were observed. After the PF reaction continued for $120 \mathrm{~h}$, the GNRs almost disappeared, with only a small amount of nanoscale fragments and debris observed under TEM (Figure 4C,F). SAED patterns reveal a severe perturbation of the graphite structure at the end of the PF reaction (insets of Figure 4C,F), which supports the nearly complete degradation of the GNRs. Furthermore, the average Raman analyses reveal that the characteristic D and $G$ signals for both samples almost disappear, suggesting full degradation of the GNRs (Figure 4G,H, left side). The 2D G band mapping shows that the $\mathrm{G}$ band intensities of both GNR samples significantly decrease over a large area after 
UV irradiation for 120 hours in comparison to the control samples before the UV irradiation ( $\mathrm{t}=0 \mathrm{~h}$ ) (Figure $4 \mathrm{G}, \mathrm{H}$, right side), while the almost negligible $\mathrm{D} / \mathrm{G}$ ratio in the $\mathrm{D} / \mathrm{G}$ band area mapping is attributed to the disappearance of the $\mathrm{D}$ and $\mathrm{G}$ bands (Figure S4). These 2D Raman mapping results confirm the high degree of degradation of the GNRs.

Further, we analyzed the possible products after the degradation of the GNRs via the PF reaction, by XPS and ESI-MS measurements. XPS analyses (Figure S5) revealed that the oxygen content of the degraded products rises significantly in the survey spectra compared to that of the control sample, meanwhile the intensities of the peaks at 286.4 $\mathrm{eV}$, which are attributed to carbon-oxygen bonds (e.g. epoxy, alkoxy, and hydroxyl groups), also clearly increase. In the MS spectra of the GNR samples treated by PF reaction for $120 \mathrm{~h}$ (Figure S6A), many peaks are detected in the $m / z$ range of $100-800$ Da. This result is similar to that reported in the previous studies on PF reaction induced degradation of graphene oxide. ${ }^{[4]}$ Based on the MS results, we have reported some possible molecular structures associated the FP degradation products, including oxidized polycyclic aromatic hydrocarbons and small molecules rich in hydroxyl, and carboxyl groups (due to the complexity of the structures, it is impossible to get a complete list of the degraded products) (Figure S6B).

Finally, we compared the degradation mechanisms of the two different protocols (Figure S7). In the case of hMPO degradation cycle, the heme group (Fe(III) protoporphyrin IX) is the active site of the enzyme (Figure S7A). The heme group stays at ferric resting state when the enzyme is not activated. Hydrogen peroxide can 

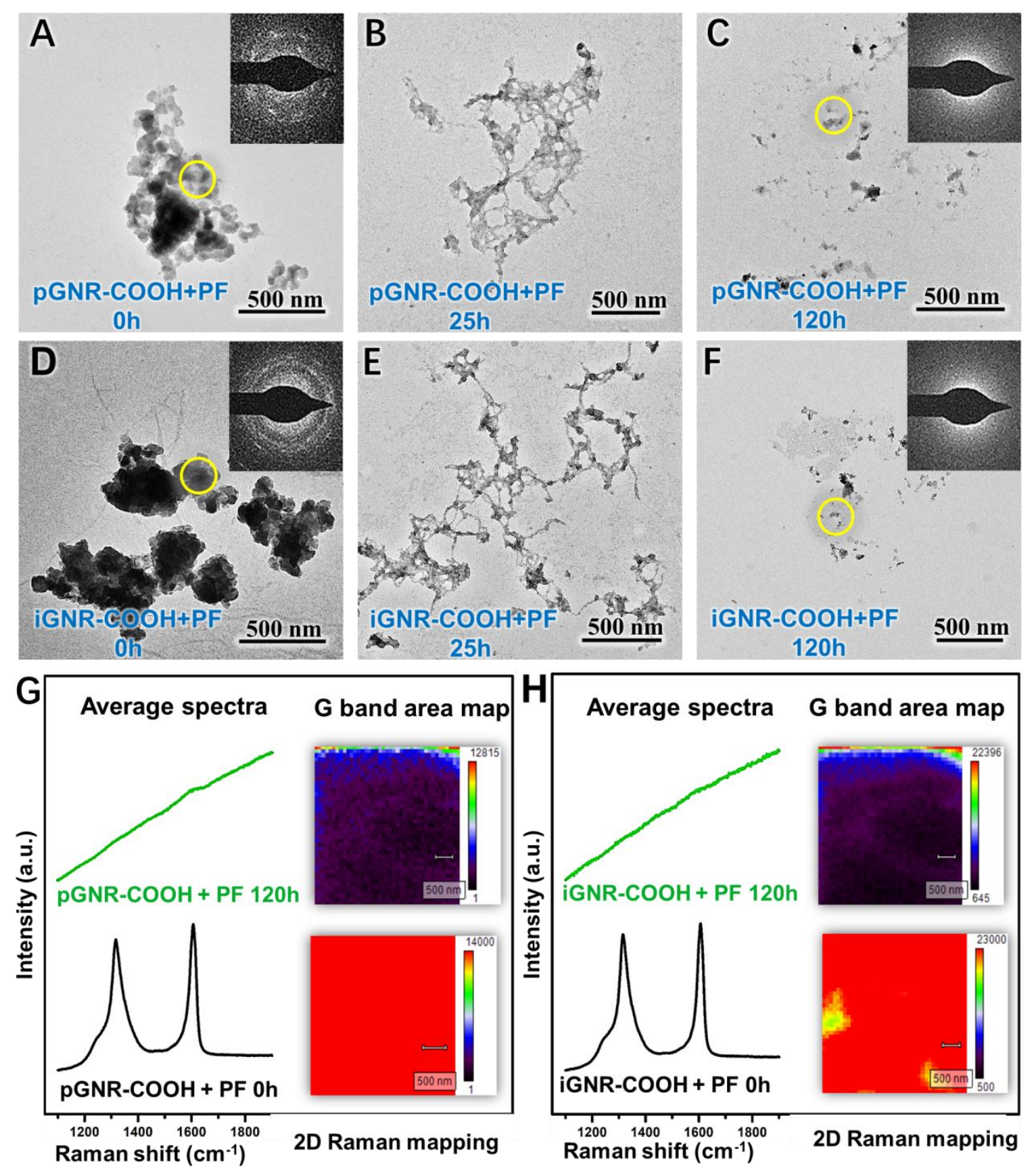

Figure 4. TEM images of the GNRs treated by UV-assisted photo-Fenton reaction at different time points: $\mathrm{t}=0 \mathrm{~h}$ (control), $\mathrm{t}=25 \mathrm{~h}$, and $\mathrm{t}=120 \mathrm{~h}$; images A-C correspond to the pGNR-COOH samples, D-F correspond to i-GNR-COOH. $(\mathrm{G}, \mathrm{H})$ Average Raman spectra (left side) and $\mathrm{G}$ band area mapping (right side) of pGNR-COOH and iGNR$\mathrm{COOH}$ treated with photo-Fenton reaction.

effectively activate hMPO, converting the heme to a high valency ferryl oxoiron $\left(\mathrm{Fe}^{4+}=\mathrm{O}\right)$ porphyrin $\pi$ cation radical (Compound I in Figure S7A). The high-valent iron in Compound I is reduced by two successive single electron transfer steps, generating a transient intermediate (Compound II), eventually returning to the resting state. In the presence of $\mathrm{Cl}^{-}$, hMPO also catalyzes the production of the strong oxidant $\mathrm{HClO}$. The 
oxidants produced during these processes, which constantly attack the GNRs, contribute to an increase in surface defects of GNRs and damages to the original clusters. ${ }^{[52]}$ The degree of degradation is closely related to the redox potential $\left(\mathrm{E}^{\mathrm{O}}\right)$ of the active intermediate produced during the oxidation process. The redox potentials of compound I and II participating in the hMPO enzymatic cycle are 1.16 and $1.34 \mathrm{~V}$, respectively. Although hMPO can produce a stronger oxidant $\mathrm{HOCl}\left(\mathrm{E}^{\mathrm{o}}=1.48 \mathrm{~V}\right)$, its redox potential is still lower than the hydroxyl radical generated by the $\mathrm{PF}$ reaction $\left(\mathrm{E}^{\mathrm{o}}=2.31 \mathrm{~V}\right.$, Figure S7B). Therefore, the PF reaction exhibits a more powerful degradation capability on GNRs compared to the natural peroxidase treatment.

In summary, carboxyl-functionalized GNRs with a well-defined structure were well dispersed in aqueous phase with the assistance of potassium hydroxide or imidazole. With good dispersibility, we demonstrated that the GNRs could be significantly degraded by both hMPO and PF reaction. Given that hMPO can be secreted by the primary immune cells, obvious biodegradation provides opportunities for potential applications of GNRs in the biological field, although the assessment of the potential toxicity of the degradation products requires further studies. Meanwhile, the PF reaction showed more effective damage to GNRs, leading to an almost complete degradation, due to the powerful oxidizing ability of the hydroxyl radicals. This offers a reliable strategy for eliminating waste GNRs accumulated in the environment.

\section{Supporting Information}

Supporting Information, including some additional figures, is available from the website or from the authors. 


\section{Acknowledgements}

We appreciate the Instrumental Analysis Center at Shanghai Jiao Tong University for some analyses. We are grateful for the financial support from the National Natural Science Foundation of China (21774076), the Program of the Shanghai Committee of Science and Technology (17JC1403200), the Program of Shanghai Academic Research Leader (19XD1421700), and the Program of Distinguished Professor of Special Appointment at Shanghai Institutions of Higher Learning. We also gratefully acknowledge the Centre National de la Recherche Scientifique (CNRS), the International Center for Frontier Research in Chemistry (icFRC), and financial support from the Agence Nationale de la Recherche (ANR) through the LabEx project Chemistry of Complex Systems (ANR-10-LABX-0026_CSC) and from European Union's Horizon 2020 research and innovation programme under the Marie Sklodowska-Curie grant agreement no. 734381 (CARBO-IMmap), We wish to thank Cathy Royer and Valérie Demais for help with TEM analyses at the "Plateforme Imagerie in vitro" at the Center of Neurochemistry (INCI, Strasbourg, France). 


\section{Reference}

[1] Y. Liu, X. Dong, P. Chen, Chem. Soc. Rev. 2012, 41, 2283-2307.

[2] B. Fadeel, C. Bussy, S. Merino, E. Vázquez, E. Flahaut, F. Mouchet, L. Evariste, L. Gauthier, A. J. Koivisto, U. Vogel, C. Martín, L. G. Delogu, T. Buerki-Thurnherr, P. Wick, D. Beloin-Saint-Pierre, R. Hischier, M. Pelin, F. C. Carniel, M. Tretiach, F. Cesca, F. Benfenati, D. Scaini, L. Ballerini, K. Kostarelos, M. Prato, A. Bianco, ACS Nano 2018, 12, 10582-10620.

[3] C. Martín, A. Ruiz, S. Keshavan, G. Reina, D. Murera, Y. Nishina, B. Fadeel, A. Bianco, Adv. Funct. Mater. 2019, 29, 1901761.

[4] A. Gazzi, L. Fusco, M. Orecchioni, S. Ferrari, G. Franzoni, J. S. Yan, M. Rieckher, G. Peng, M. A. Lucherelli, I. A. Vacchi, N. D. Q. Chau, A. Criado, A. Istif1, D. Mancino, A. Dominguez, H. Eckert, E. Vazquez, T. Da Ros, P. Nicolussi, V. Palermo, B. Schumacher, G. Cuniberti, Y. Mai, C. Clementi, M. Pasquali, X. Feng, K. Kostarelos, A. Yilmazer, D. Bedognetti, B. Fadeel, M. Prato, A. Bianco, L. G. Delogu, J. Phys. Mater 2020, doi:10.1088/2515-7639/ab9317.

[5] J. Cai, P. Ruffieux, R. Jaafar, M. Bieri, T. Braun, S. Blankenburg, M. Muoth, A. P. Seitsonen, M. Saleh, X. Feng, K. Müllen, R. Fasel, Nature 2010, 466, 470-473.

[6] D. J. Rizzo, V. Gregory, T. Cao, B. Christopher, T. Chen, F. Zhao, R. Henry, S. G. Louie, M. F. Crommie, F. R. Fischer, Nature 2018, 560, 204-208.

[7] O. Gröning, S. Wang, X. Yao, C. A. Pignedoli, G. B. Barin, C. Daniels, A. Cupo, V. Meunier, X. Feng, Narita, K. Müllen, P. Ruffieux, R. Fasel, Nature 2018, 560, 209213.

[8] G. D. Nguyen, H. Tsai, A. A. Omrani, T. Marangoni, M. Wu, D. J. Rizzo, G. F. Rodgers, R. R. Cloke, R. A. Durr, Y. Sakai, F. Liou, A. S. Aikawa, J. R. Chelikowsky, S. G. Louie, F. R. Fischer, M. F. Crommie, Nat. Nanotechnol. 2017, 12, 1077-1082.

[9] A. Narita, X. Feng, Y. Hernandez, S. A. Jensen, M. Bonn, H. Yang, I. A. Verzhbitskiy, C. Casiraghi, M. R. Hansen, A. H. R. Koch, G. Fytas, O. Ivasenko, B. Li, K. S. Mali, T. Balandina, S.Mahesh, S. D. Feyter, K. Müllen, Nat. Chem. 2014, $6,126-132$.

[10]O. Deniz, C. Sánchez-Sánchez, T. Dumslaff, X. Feng, A. Narita, K. Müllen, N. Kharche, V. Meunier, R. Fasel, P. Ruffieux, Nano Lett. 2017, 17, 2197-2203.

[11]T. H. Vo, M. Shekhirev, D. A. Kunkel, M. D. Morton, E. J. Berglund, L. Kong, P. M. Wilson, P. A. Dowben, A. Enders, A. Sinitskii, Nat. Commun. 2014, 5, 3189. 
[12]Y. Yano, F. Wang, N. Mitoma, Y. Miyauchi, H. Ito, K. Itami, J. Am. Chem. Soc. 2020, $142,1686-1691$.

[13] Y. Yano, N. Mitoma, K. Matsushima, F. Wang, K. Matsui, A. Takakura, Y. Miyauchi, H. Ito, K. Itami, Nature 2019, 571, 387-392.

[14]G. Li, K. Y. Yoon, X. Zhong, J. Wang, R. Zhang, J. R. Guest, J. Wen, X. Y. Zhu, G. Dong, Nat. Commun. 2018, 9, 1687.

[15] Y. Yano, N. Mitoma, H. Ito, K. Itami, J. Org. Chem. 2020, 85, 4-33.

[16]A. Jolly, D. Miao, M. Daigle, J. Morin, Angew. Chem. Int. Ed. 2020, 59, 4624-4633; Angew. Chem. 2020, 132, $4652-4661$

[17] Y. Huang, F. Xu, L. Ganzer, F. V. A. Camargo, T. Nagahara, J. Teyssandier, H. Van Gorp, K. Basse, L. A. Straaso, V. Nagyte, C. Casiraghi, M. R. Hansen, S. D. Feyter, D. Yan, K. Müllen, X. Feng, G. Cerullo, Y. Mai, J. Am. Chem. Soc. 2018, 140, 10416-10420.

[18]W. Niu, J. Liu, Y. Mai, K. Müllen, X. Feng, Trends in Chemistry 2019, 1, 549-558.

[19]F. Xu, J. Zhang, P. Zhang, X. Luan, Y. Mai, Mater. Chem. Front. 2019, 3, 22832307.

[20]Y. Huang, Y. Mai, U. Beser, J. Teyssandier, G. Velpula, H. Van Gorp, L. A. Straaso, M. R. Hansen, D. Rizzo, C. Casiraghi, R. Yang, G. Zhang, D. Wu, F. Zhang, D. Yan, S. D. Feyter, K. Müllen, X. Feng, J. Am. Chem. Soc. 2016, 138, 10136-10139.

[21]Y. Huang, W. Dou, F. Xu, H. Ru, Q. Gong, D. Wu, D. Yan, H. Tian, X. He, Y. Mai, X. Feng, Angew. Chem. Int. Ed. 2018, 57, 3366-3371; Angew. Chem. 2018, $130,3424-3429.2$

[22]Z. Yu, X. Li, F. Xu, X. Hu, J. Yan, N. Kwon, G. Chen, T. Tang, X. Dong, Y. Mai, D. Chen, J. Yoon, X. He, He Tian, Angew. Chem. Int. Ed. 2020, 59, 3658-3664; Angew. Chem. 2020, 132, 3687 - 3693.

[23]D. Joshi, M. Hauser, G. Veber, A. Berl, K. Xu, F. R. Fischer, J. Am. Chem. Soc. 2018, 140, 9574-9580.

[24]M. Y. Han, B. Oezyilmaz, Y. Zhang, P. Kim, Phys. Rev. Lett. 2007, 98, 206805.

[25]J. G. Son, M. Son, K. J. Moon, B. H. Lee, J. M. Myoung, M. S. Strano, M. Ham, C. A. Ross, Adv. Mater. 2013, 25, 4723-4728.

[26] V. Passi, A. Gahoi, B. V. Senkovskiy, D. Haberer, F. R. Fischer, A. Gruneis, M. C. Lemme, ACS Appl. Mater. Interfaces 2018, 10, 9900-9903.

[27]C. Rogers, W. S. Perkins, G. Veber, T. E. Williams, R. R. Cloke, F. R. Fischer, J. Am. Chem. Soc. 2017, 139, 4052-4061. 
[28]H. Shen, A. Cresti, W. Escoffier, Y. Shi, X. Wang, B. Raquet, ACS Nano 2016, 10, 1853-1858.

[29]A. N. Abbas, G. Liu, A. Narita, M. Orosco, X. Feng, K. Müllen, C. Zhou, J. Am. Chem. Soc. 2014, 136, 7555-7558.

[30]D. Krepel, J. E. Peralta, G. E. Scuseria, O. Hod, J. Phys. Chem. C 2016, 120, 37913797.

[31]M. M. Pour, A. Lashkov, A. Radocea, X. Liu, T. Sun, A. Lipatov, R. Korlacki, M. Shekhirev, N. R. Aluru, J. W. Lyding, V. Sysoev, A. Sinitskii, Nat. Commun. 2017, $8,820-820$.

[32]M. C. Chong, N. Afshar-Imani, F. Scheurer, C. Cardoso, A. Ferretti, D. Prezzi, G. Schull, Nano Lett. 2018, 18, 175-181.

[33]Y. Huang, Y. Mai, X. Yang, U. Beser, J. Liu, F. Zhang, D. Yan, K. Müllen, X. Feng, J. Am. Chem. Soc. 2015, 137, 11602-11605.

[34]D. Wu, F. Xu, Y. Huang, C. Chen, C. Yu, X. Feng, D. Yan, Y. Mai, Macromolecules 2018, 51, 161-172.

[35]F. Xu, C. Yu, A. Tries, H. Zhang, M. Klaui, K. Basse, M. R. Hansen, N. Bilbao, M. Bonn, H. I. Wang, Y. Mai, J. Am. Chem. Soc. 2019, 141, 10972-10977

[36]G. Veber, C. S. Diercks, C. Rogers, W. S. Perkins, J. Ciston, K. Lee, J. P. Llinas, A. Liebmanpelaez, C. Zhu, J. Bokor, F. R. Fischer, Chem 2020. 6, 1125-1133

[37]A. Tries, S. Osella, P. Zhang, F. Xu, C. Ramanan, M. Kläui, Y. Mai, D. Beljonne, H. I. Wang, Nano Lett. 2020, 20, 2993-3002.

[38]B. L. Allen, G. P. Kotchey, Y. Chen, N. Yanamala, J. Kleinseetharaman, V. E. Kagan, A. Star, J. Am. Chem. Soc. 2009, 131, 17194-17205.

[39]R. Kurapati, J. Russier, M. A. Squillaci, E. Treossi, C. Ménard-Moyon, A. E. D. Riocastillo, E. Vazquez, P. Samorì, V. Palermo, A. Bianco, Small 2015, 11, 39853994.

[40]R. Kurapati, S. P. Mukherjee, C. Martín, G. Bepete, E. Vazquez, A. Penicaud, B. Fadeel, A. Bianco, Angew. Chem. Int. Ed. 2018, 57, 11722-11727; Angew.Chem. 2018, 130, 11896-11901.

[41]C. Martín, G. Jun, R. Schurhammer, G. Reina, P. Chen, A. Bianco, C. MénardMoyon, Small 2019, 15, 1905405.

[42]R. Kurapati, C. Martín, V. Palermo, Y. Nishina, A. Bianco, Faraday Discuss. 2020, 10.1039/C9FD00094A.

[43]G. Lalwani, W. Xing, B. Sitharaman, J. Mater. Chem. B 2014, 2, 6354-6362. 
[44]X. Zhou, Y. Zhang, C. Wang, X. Wu, Y. Yang, B. Zheng, H. Wu, S. Guo, J. Zhang, ACS Nano 2012, 6, 6592-6599.

[45]H. Bai, W. Jiang, G. P. Kotchey, W. A. Saidi, B. J. Bythell, J. M. Jarvis, A. G. Marshall, R. A. S. Robinson, A. Star, J. Phys. Chem. C 2014, 118, 10519-10529.

[46]D. Spuhler, J. A. Rengifo-Herrera, C. Pulgarin, Appl. Catal. B 2010, 96, 126-141.

[47]S. Navalon, A. Dhakshinamoorthy, M. Alvaro, M. Antonietti, H. Garcia, Chem. Soc. Rev. 2017, 46, 4501-4529.

[48]R. Kurapati, C. Backes, C. Ménard-Moyon, J. N. Coleman, A. Bianco, Angew. Chem. Int. Ed. 2016, 55, 5506-5511; Angew.Chem. 2016, 128, 5596-5601.

[49]P. Chen, Y. Chen, N. Kao, C. Wu, Y. Zhang, H. Li, Carbon 2015, 90, 16-24.

[50]H. K. Jeong, Y. P. Lee, R. J. W. E. Lahaye, M. Park, K. H. An, I. J. Kim, C. Yang, C. Y. Park, R. S. Ruoff, Y. H. Lee, J. Am. Chem. Soc. 2008, 130, 1362-1366.

[51]G. P. Kotchey, B. L. Allen, H. Vedala, N. Yanamala, A. A. Kapralov, Y. Y. Tyurina, J. Kleinseetharaman, V. E. Kagan, A. Star, ACS Nano 2011, 5, 2098-2108.

[52] G. P. Kotchey, S. A. Hasan, A. A. Kapralov, S. H. Ha, K. Kim, A. A. Shvedova, V. E. Kagan, A. Star, Acc. Chem. Res. 2012, 45, 1770-1781. 